\title{
BMJ Open Development of a self-guided web- based exercise intervention (SPIN) to treat shoulder pain in people living with spinal cord injury: protocol of a mixed methods study
}

\author{
Verna Stavric, ${ }^{\oplus 1}$ Nicola Saywell, ${ }^{1}$ Nicola Maree Kayes ${ }^{2}$
}

To cite: Stavric V, Saywell N Kayes NM. Development of a self-guided web-based exercise intervention (SPIN) to treat shoulder pain in people living with spinal cord injury: protocol of a mixed methods study. BMJ Open 2019;9:e031012. doi:10.1136/ bmjopen-2019-031012

- Prepublication history for this paper is available online. To view these files, please visit the journal online (http://dx.doi org/10.1136/bmjopen-2019031012).

Received 12 April 2019 Revised 03 August 2019 Accepted 22 August 2019

Check for updates

(C) Author(s) (or their employer(s)) 2019. Re-use permitted under CC BY-NC. No commercial re-use. See rights and permissions. Published by BMJ.

${ }^{1}$ Physiotherapy, School of Clinical Sciences, Auckland University of Technology, Auckland, New Zealand ${ }^{2}$ Centre for Person Centred Research, School of Clinical Sciences, Auckland University of Technology, Auckland, New Zealand

Correspondence to

Verna Stavric;

verna.stavric@aut.ac.nz

\section{ABSTRACT}

Introduction Chronic shoulder pain is common after spinal cord injury (SCl) and limits community mobility. This leads to loss of independence and reduced quality of life. Evidence suggests that exercises can help reduce shoulder pain. However, cost, expertise and transport barriers frequently limit access to treatment services. The objective of this study is to develop an evidence-based, acceptable, usable and persuasive self-guided web-based exercise intervention to treat shoulder pain in people living with $\mathrm{SCl}$. Methods and analysis An iterative and phased personbased approach (PBA) will capture users' perspectives on usability and acceptability to develop guiding principles that will shape the design of the intervention. The intervention will be based on key elements identified through participant input and from evidence identified through systematic and narrative reviews, to ensure the intervention addresses participants' needs and increase the likelihood of uptake. The prototype will be iteratively refined through focus groups and think-aloud sessions. Review data will be synthesised drawing on systematic and narrative review conventions. Qualitative data will be analysed using conventional content analysis (planning phase) and directed content analysis (development phase) to inform intervention design and refinement.

Ethics and dissemination Ethical approval has been granted by the Auckland University of Technology Ethics Committee (AUTEC) in Auckland, New Zealand. The results of the study will be published in a peer-reviewed journal and presented at relevant national and international conferences. A summary of findings will be presented to key stakeholder groups. We will progress to a definitive trial should the findings from this intervention development study indicate the intervention is acceptable and usable.

\section{INTRODUCTION}

People with spinal cord injury or spinal cord impairment (from non-traumatic causes) (SCI) rely on their upper extremities for locomotion as well as performance of daily activities. Consequently, up to $70 \%$ of people living with SCI experience shoulder pain, which can have a significant impact on activity that
Strengths and limitations of this study

- Using the perspectives of potential users in intervention design drawing on the person-based approach may increase the likelihood of engagement and usability.

- Having a stakeholder advisory group has the potential to support translation of the findings to real-world application.

- Budgetary restraints may limit the degree of intervention refinement possible, which may slow development of the final product.

reduces community mobility, independence and quality of life. ${ }^{1-6}$

Exercise-based rehabilitation is often included in the management of shoulder pain. Protocols including stretches and strengthening exercises have been shown to significantly reduce shoulder pain in people with SCI in a series of non-controlled studies, ${ }^{7-10}$ a randomised controlled study ${ }^{11}$ and a systematic review. ${ }^{12}$ Despite the known benefits of exercise to reduce shoulder pain, many people living with SCI who experience shoulder pain often do not engage in these exercises. ${ }^{13}$ They cite barriers to accessing exercise and rehabilitation opportunities that include limited access to knowledgeable health professionals, poor physical accessibility and transportation difficulties. $^{14} 15$ Digital health interventions offer a potential opportunity to overcome many of these barriers in a cost-effective way. ${ }^{16}$ They can provide automated and remote personalised feedback and support for selfguided exercise, in a person's own time and environment.

Although web-based exercise resources are currently available for people living with SCI, ${ }^{17} 18$ they have some limitations. For 


\begin{tabular}{|c|c|c|c|}
\hline \multirow[t]{2}{*}{ PBA Description } & \multirow[t]{2}{*}{ Phase } & \multicolumn{2}{|c|}{ Current Study } \\
\hline & & Purpose & Planned Outcome \\
\hline $\begin{array}{l}\text { Use of primary and } \\
\text { secondary qualitative } \\
\text { evidence to understand } \\
\text { users' behavioural and } \\
\text { psychosocial needs and } \\
\text { challenges in using the } \\
\text { intervention. }\end{array}$ & Planning & $\begin{array}{l}\text { To determine factors that } \\
\text { need to be included to } \\
\text { encourage or facilitate } \\
\text { engagement with this self- } \\
\text { guided web-based exercise } \\
\text { intervention. }\end{array}$ & $\begin{array}{l}\text { A rich description of key needs, } \\
\text { challenges and facilitators of } \\
\text { engagement in web-based tools } \\
\text { and exercise for people living } \\
\text { with } \mathrm{SCl} \text { who experience } \\
\text { shoulder pain to underpin the } \\
\text { design phase's guiding } \\
\text { principles and features. }\end{array}$ \\
\hline $\begin{array}{l}\text { Formulation of key guiding } \\
\text { principles that capture the } \\
\text { main intervention objectives } \\
\text { as identified in the planning } \\
\text { phase and that are } \\
\text { continuously referred to } \\
\text { throughout the } \\
\text { development of the } \\
\text { intervention. }\end{array}$ & Design & $\begin{array}{l}\text { To design an evidence- } \\
\text { based, self-guided, web- } \\
\text { based intervention. } \\
\text { Exercise, behavioural } \\
\text { support and self-guided } \\
\text { components will be included } \\
\text { within the intervention } \\
\text { features. }\end{array}$ & $\begin{array}{l}\text { Key intervention objectives. } \\
\text { Key intervention features. } \\
1^{\text {st }} \text { iteration of SPIN prototype } \\
\text { (wireframe version). }\end{array}$ \\
\hline $\begin{array}{l}\text { Intervention components } \\
\text { are evaluated and optimised } \\
\text { based on user feedback. }\end{array}$ & Development & $\begin{array}{l}\text { To develop an evidence- } \\
\text { based, self-guided, web- } \\
\text { based intervention. } \\
\text { Iterative feedback and } \\
\text { prototyping to refine the } \\
\text { intervention through } \\
\text { wireframe focus group } \\
\text { testing and then further } \\
\text { refined with think-aloud } \\
\text { testing. }\end{array}$ & $\begin{array}{l}\text { Final SPIN prototype } \\
\text { (digital version). } \\
\text { Ready for trialling. }\end{array}$ \\
\hline $\begin{array}{l}\text { Intervention is evaluated in } \\
\text { real-life context and } \\
\text { modified to improve future } \\
\text { implementation. }\end{array}$ & Trialling & $\begin{array}{l}\text { To carry out a mixed } \\
\text { methods pilot trial. This } \\
\text { phase will be dependent on } \\
\text { the preceding phases. }\end{array}$ & $\begin{array}{l}\text { A brief outline entitled 'future } \\
\text { work' is included in this paper. }\end{array}$ \\
\hline
\end{tabular}

Figure 1 Phases of intervention development. PBA, person-based approach; SCI, spinal cord injury; SPIN, Shoulder Pain Intervention delivered over the interNet.

example, they require the ongoing support of a clinician, are not specific to treating shoulder pain or do not have the capability to automate exercise progression. Shoulder Pain Intervention delivered over the interNet (SPIN) is being developed to address these limitations. To our knowledge, this will be the first web-based, self-guided intervention that will prescribe, monitor and progress evidence-based exercises for people living with SCI who experience shoulder pain. The intervention will be an interactive tool using responses from users on their pain or degree of exercise difficulty to tailor the programme.

Translation of an existing evidence-based intervention into a web-based format presents a number of challenges. ${ }^{19}$ For example, attracting users and encouraging engagement with the intervention can be further complicated by how usable the technology is and how quickly it continues to evolve. Therefore, the development of SPIN will be theory-driven, evidence-based and underpinned by the person-based approach (PBA) to intervention development. ${ }^{20}$ The PBA seeks a deep understanding of the perspectives and psychosocial context of potential users through iterative qualitative research. ${ }^{20}$ The PBA draws on evidence from primary and secondary sources to identify barriers and facilitators to uptake. As Yardley et $a l^{20}$ suggest, it makes use of behavioural evidence and theory, ${ }^{21}$ while keeping the user's needs and context in focus, increasing the likely engagement in and effectiveness of the intervention. ${ }^{22-24}$ SPIN is planned to be self-guided and so will be used with minimal health professional contact. As such, ensuring the design is underpinned by a clear understanding of the perceptions, assumptions, behavioural needs and challenges of the user will increase its relevance and usability. The aim of this project is to develop an evidence-based, acceptable, usable and persuasive self-guided web-based exercise intervention to treat shoulder pain in people living with SCI.

\section{METHODS AND ANALYSIS}

\section{Patient and public involvement}

The research question was developed from clinical experiences and then further refined through consultation with the Burwood Academy of Independent Living End User Consultation Committee, a consumer group with 
the expertise of living with SCI who advises on research projects. $^{25}$

A stakeholder advisory group (SAG; more details are provided below) will also be formed to help support the study at key points. Key findings will be presented by researchers and participants at community and academic meetings.

\section{Study design}

The PBA proposes four phases of intervention development that include planning, design, development and trialling. The current study is focused primarily on the first three phases. Figure 1 provides a definition of each phase according to the PBA and an overview of how they map onto the current study. The iterative nature of intervention development implies that phases are not discreet, and movement will occur between them.

\section{Participants \\ Eligibility}

Participants will be eligible if they reside in New Zealand; are living with SCI; have completed active rehabilitation; are over 16 years of age; have capacity to give informed consent; are predominantly wheelchair users; and are experiencing or have recently (within 2 years) experienced shoulder pain. Participants will be excluded if they are unable to communicate with the researcher for the purposes of meaningful engagement in data collection.

\section{Recruitment}

Posters will be distributed within SCI community networks and SCI services and rehabilitation providers. Information will also be circulated through social media sites and professional and personal networks. People who express interest in the study will be invited to contact the researcher or give permission for the researcher to contact them. The sampling approach specific to each phase is provided in more detail below.

\section{Stakeholder advisory group}

An SAG will be formed to support this project. The composition of the group will include a person living with SCI, a clinician with experience in SCI rehabilitation, a representative of a relevant non-governmental organisation and a computer engineer with knowledge in web design and decision tree development. They will meet at least four times during the study including at the outset and following each phase. Their primary role will be to gauge how findings resonate with personal insights and experience, to make recommendations for the subsequent phases and to inform refinements to the intervention. For example, in the planning phase, they will be able to make recommendations for recruitment.

\section{Planning phase: collecting and synthesising evidence Purpose}

- To determine the effectiveness of existing self-guided web-based exercise interventions.
- To determine factors that need to be included to facilitate engagement with the current self-guided web-based exercise intervention.

This phase will include collecting and synthesising primary and secondary evidence to inform the design of the intervention. Secondary evidence will include both a systematic and narrative review of the literature. Primary evidence will be collected through an interpretive descriptive study.

Systematic review of effectiveness of self-guided web-based exercise interventions

Yardley et $a t^{20}$ recommend drawing on an existing evidence base. However, there are no reviews currently available that synthesise evidence regarding the effectiveness of self-guided, web-based exercise interventions. Consequently, the first stage in this work is to conduct a systematic review to (1) determine effectiveness of this method of delivery in improving health outcomes for those with chronic health conditions and (2) extract data on key characteristics of those interventions identified as effective. The results will be used to inform intervention development by identifying elements common to effective web-based interventions.

The systematic review will follow the Preferred Reporting Items for Systematic Reviews and Meta-Analyses (PRISMA) statement. ${ }^{26}$

\section{Eligibility criteria}

Studies will be included if they meet the inclusion and exclusion criteria presented in table 1. Initial scoping revealed no applicable interventions involving people living with SCI. Therefore, the search was expanded to include those living with a chronic health condition.

\section{Databases}

Literature searches will be conducted in the following databases: Cumulated Index to Nursing and Allied Health Literature (CINAHL), MEDLINE, SPORTSDiscus through EBSCO Health Database, Allied and Complementary Medicine (AMED), Evidence-Based Medicine (EBM) Reviews-Cochrane Methodology Register third Quarter 2012, EBM Reviews-Health Technology Assessment fourth Quarter 2016 PsycINFO 1806 to July Week 2, 2017, MEDLINE(R) Epub Ahead of Print, In-Process and Other Non-Indexed Citations, Ovid MEDLINE(R) Daily and Ovid MEDLINE(R) 1946 to present through OVID, in Scopus and in Web of Science. The Physiotherapy Evidence Database (PEDro) will be searched (using simplified broad key terms) as a checking exercise. Reference lists of relevant reviews and studies will be hand searched.

A search strategy has been devised drawing on the Population, Intervention, Comparison, Outcome (PICO) framework with a focus on key terms relevant to intervention (web-based exercise) and study design (randomised control trial). The search will not be limited by population and outcome to keep the reach as broad as possible and ensure 
Table 1 Inclusion/exclusion criteria for systematic review

\begin{tabular}{lll}
\hline Elements & Inclusion criteria & Exclusion criteria \\
\hline Study design and & Randomised controlled trial or pilot that contains data addressing effectiveness & Not in English \\
reporting & Full text available & Publication not peer- \\
& & reviewed \\
& & Conference \\
& proceeding
\end{tabular}

$\begin{array}{ll}\text { Population } & \text { Adults with an existing chronic health condition } \\ \text { Intervention } & \text { Designed for the use of people living with a chronic health condition } \\ & \text { Explicitly supports self-guided physical activity or exercise in a self-guided } \\ \text { programme } & \text { Web-based or app-based } \\ & \begin{array}{l}\text { Intervention has minimal human contact comprising no more than initial contact } \\ \text { for set up or orientation. Has ongoing contact that is generated automatically. }\end{array} \\ \text { Outcome } & \text { Health related }\end{array}$

studies are not missed. Search strategies will also include Boolean, wildcard, truncation and proximity searching, tailored by database. Table 2 provides an example of a search to be conducted using an OVID database.

\section{Selection of studies}

All citations returned in the search will be downloaded and saved into EndNote X8. Duplicates will be removed and then titles will be screened by VS, according to the predefined inclusion criteria. Initially, a selection of titles will be independently screened by a second assessor (NS). Any disagreements will be reviewed and discussed to ensure consensus is reached. Should agreement not be reached, a third assessor (NMK) will serve as arbitrator. The abstracts and then full texts of all those studies potentially meeting the inclusion criteria will be reviewed by VS before settling on a final set of included studies in consultation with NS.

Data extraction and management

Key details from each of the included studies will be recorded in data extraction tables. Details will include author and country, study design, participant numbers and characteristics, treatment intervention (including features and components used) and health outcomes.

\section{Quality assessment}

Risk of bias for each of the included studies will be assessed as low, high or unclear drawing on guidelines by The Cochrane Collaboration's tool for assessing risk of bias in randomised trials. ${ }^{27}$ Appraisal of the quality of included studies will follow the criteria outlined in the Critical Appraisal Skills Programme Randomised Controlled Trial Checklist. ${ }^{28}$

\section{Narrative review of relevant literature}

The planning phase includes using qualitative evidence to inform intervention development. This second review of literature will explore what helps or hinders engagement with (1) exercise and physical activity and (2) web-based interventions for people living with SCI. The findings will be used to generate discussion topics for the Interpretive descriptive study (described further below). Findings will also inform the guiding principles for intervention design.

\begin{tabular}{|c|c|c|}
\hline & Search concept & Likely terms \\
\hline$\# 1$ & Web-based exercise & $\begin{array}{l}\text { (web* ADJ6 Exercis*) OR (web* ADJ6 Rehabilitation) OR (web* ADJ6 Physiotherap*) OR } \\
\text { (web* ADJ6 'Physical therap*') OR (web* ADJ6 therap*) OR (web* ADJ6 'fitness training') } \\
\text { OR (web* ADJ6 physical activit*) OR (internet* ADJ6 Exercis*) OR (internet* ADJ6 } \\
\text { Rehabilitation) OR (internet* ADJ6 Physiotherap*) OR (internet ADJ6 'Physical therap*') } \\
\text { OR (internet* ADJ6 therap*) OR (internet* ADJ6 'fitness training') OR (internet* ADJ6 } \\
\text { physical activit*) OR (online ADJ6 Exercis*) OR (online ADJ6 Rehabilitation) OR (online } \\
\text { ADJ6 Physiotherap*) OR (online* ADJ6 'Physical therap*') OR (online ADJ6 therap*) OR } \\
\text { (online* ADJ6 'fitness training') OR (online ADJ6 physical activit*) }\end{array}$ \\
\hline \#2 & $\begin{array}{l}\text { E-health or } \\
\text { physiotherapy }\end{array}$ & 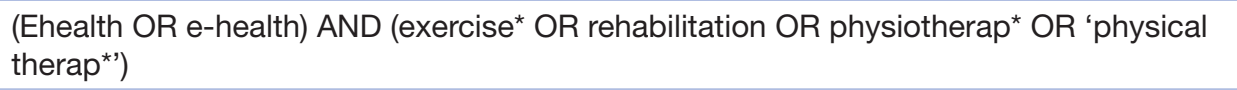 \\
\hline \#3 & \#1 OR \#2 & \\
\hline
\end{tabular}


Table 3 Inclusion/exclusion criteria for narrative review

\begin{tabular}{lll}
\hline Elements & Inclusion criteria & Exclusion criteria \\
\hline $\begin{array}{ll}\text { Study design and formatting } \\
\text { Phenomena of interest }\end{array}$ & Qualitative study & Not in English \\
& $\begin{array}{l}\text { Full text available } \\
\text { Exploring experiences and perspectives of physical activity }\end{array}$ & $\begin{array}{l}\text { Publication not peer- } \\
\text { reviewed } \\
\text { interventions }\end{array}$ \\
& $\begin{array}{l}\text { Exploring experiences and perspectives of web-based } \\
\text { interventions }\end{array}$ & \\
Participants & Adults living with $\mathrm{SCl}$ who experience mobility limitation & \\
\hline
\end{tabular}

$\mathrm{SCl}$, spinal cord injury.

\section{Eligibility criteria}

Studies will be included if they meet the inclusion and exclusion criteria outlined in table 3 .

\section{Search strategy}

Literature searches will be conducted in the following databases: Cumulated Index to Nursing and Allied Health Literature (CINAHL), MEDLINE, SPORTSDiscus through EBSCO Health Database, Allied and Complementary Medicine (AMED), Evidence-Based Medicine (EBM) Reviews-Cochrane Methodology Register third Quarter 2012, EBM Reviews-Health Technology Assessment fourth Quarter 2016, PsycINFO 1806 to July Week 2, 2017, MEDLINE(R) Epub Ahead of Print, In-Process and Other Non-Indexed Citations, Ovid MEDLINE(R) Daily and Ovid MEDLINE(R) 1946 to present through OVID, in Scopus and in Web of Science. Reference lists of relevant reviews and studies will also be searched.

A search strategy will be devised by breaking the review question down into component parts including key words relevant to patient perspective, the substantive topics (web-based intervention, exercise and physical activity) and study design. Table 4 provides an example of a strategy that combines these components and possible search terms. Searches will be run for each component and then combined to reflect the relevant question (see table 4 searches \#6 and \#7).

\section{Selection of studies}

This review will follow a similar approach to the data selection as the systematic review described earlier.

\section{Data selection and management}

Key details from each of the included studies will be recorded in data extraction tables. Details will include author details and country, study design, participant numbers and characteristics, experience explored (eg, exercise or web-based interventions), barriers to engagement and facilitators to engagement.

\section{Quality assessment}

Appraisal of the quality of included studies will follow the criteria as outlined in the Critical Appraisal Skills Programme Qualitative Checklist. ${ }^{29}$

\section{Data analysis}

A narrative synthesis of data will be performed. Findings from the included studies will be reviewed and key concepts relevant to the review questions identified. These will be grouped into categories and then mapped to the original findings of included papers to check for resonance and identify aspects that either confirm or conflict with the synthesised findings. From this, a more refined set of categories will be generated.

Table 4 Narrative review search concepts and terms

\begin{tabular}{|c|c|c|}
\hline & Search concept & Likely terms \\
\hline$\# 1$ & Experiences & $\begin{array}{l}\text { barrier }^{\star} \text { OR facilitator }{ }^{\star} \text { OR help* OR hinder* OR perspective* OR experience* OR } \\
\text { acceptability OR satisfaction OR view* OR perception* }\end{array}$ \\
\hline \#3 & $\begin{array}{l}\text { Physical activity or } \\
\text { exercise }\end{array}$ & $\begin{array}{l}\text { exercise* OR rehabilitation OR physiotherapy OR physical therapy OR therapy OR } \\
\text { 'fitness training' OR 'training' OR 'physical activity' }\end{array}$ \\
\hline \#5 & Spinal cord injury & Spinal cord injur^ OR 'SCl' OR paraplegi^ OR quadriplegi^ OR tetraplegi^ \\
\hline \#6 & $\begin{array}{l}\# 1 \text { AND \#2 AND \#3 AND } \\
\# 5\end{array}$ & \\
\hline \#7 & $\begin{array}{l}\text { \#1 AND \#2 AND \#4 AND } \\
\# 5\end{array}$ & \\
\hline
\end{tabular}

$\mathrm{SCl}$, spinal cord injury. 
Interpretive descriptive study

Primary qualitative evidence will be collected in this phase. An interpretive descriptive ${ }^{30}$ study will be carried out to explore the perceptions of people who live with SCI regarding a self-guided, web-based, exercise intervention to treat shoulder pain. The results will inform the guiding principles to underpin intervention design.

\section{Participants}

Participants (up to $n=20$ ) will be recruited using the eligibility criteria and recruitment process described earlier. We will draw on Malterud's concept of information power $^{31}$ to guide sample size. Participants will be purposively selected to ensure diversity in social circumstances, age, ethnicity, gender and impairment to ensure breadth of perspectives and experiences.

\section{Data collection}

Individual or focus group interviews will be conducted at a venue convenient to the participant(s), such as their residence, place of employment or another agreed location. Individual interviews may be carried out via other means (ie, Skype, FaceTime) if preferred by the participant. A semistructured interview guide will be used to focus on the phenomenon of interest while allowing enough flexibility for the interviewer to be responsive to participant responses. ${ }^{32}$ Topic areas will include participants' perceptions and suggestions of various web-based exercise scenarios, exploring concepts uncovered by the review findings. This will be followed by questions relating to how features of a web-based intervention could support people, for example, how it would need to look, what would encourage participants to use it and to what extent it may overcome barriers identified in the review. Opportunities for problem solving and contribution to the intervention design process will also be integrated into these sessions. ${ }^{33}$ For example, participants will be invited to comment on currently available website and app features that could be included in a future web-based intervention. Interviews will be audio recorded and transcribed verbatim.

\section{Data analysis}

Data will be analysed using conventional content analysis. ${ }^{34}$ The transcripts will be read and reread to allow familiarity with the data and to identify items of potential interest. They will be read in conjunction with researcher notes taken at the time of the interviews so that the data and its context are considered when reading. Next, data from several transcripts will be inductively coded, using exact words from the text, where possible, to capture key concepts. Memos will be used to capture the researchers' initial impressions throughout this initial coding process. The codes and relevant data will then be collated in an iterative process of returning to the original data for recoding and refinement. Initial codes and memos will subsequently be examined to identify meaningful clusters. Following this, a coding framework with associated definitions will be developed. All transcripts, memos and codes will be entered into NVivo V.12. ${ }^{35}$ The remaining transcripts will then be analysed by searching for statements that specifically support or challenge the proposed codes to refine the cluster and categorisation. Strategies to ensure robust and rigorous interpretation will be used, drawing on Thorne's notions of epistemological integrity, representative credibility, analytic logic and interpretive authority. ${ }^{36}$

\section{Design phase}

Purpose

- To develop an evidence-based web-based prototype intervention.

The design phase will synthesise information from the planning phase, in keeping with the PBA, to formulate guiding principles. These are statements that succinctly reflect what is distinctive about the intervention that meets user needs as identified in the planning phase. ${ }^{20}$ They ensure intervention development remains constant and true in its direction towards meeting the earlier identified goals. The guiding principles can be broken down into intervention objectives and the key intervention features needed to achieve them. Data will be drawn and integrated from several sources to underpin intervention objectives and features including (1) existing evidence-based exercise interventions that address shoulder pain; (2) systematic review findings regarding features of effective self-guided and web-based interventions; (3) narrative review and interpretive descriptive study findings regarding aspects that support acceptability and engagement and (4) relevant behavioural theory. For example, if synthesis reveals that valuing independence is important, then an objective of the intervention would be that it can be used autonomously. This will then inform a set of related intervention features such as customisable elements for goal setting, self-monitoring and choice.

The first iteration of the SPIN prototype will be produced during this phase as a set of wireframes. Wireframes are a paper-based visual schematic of the prototype that help participants experience working through the intervention's proposed sequence without live data or graphic design. It therefore allows researchers and participants to discuss the features of the intervention separate to the aesthetics. $^{37}$

\section{Development phase \\ Purpose}

- To develop an evidence-based web-based intervention. During the development phase, people with SCI will take part in usability testing, providing feedback on the wireframes produced in the design phase. Data will guide the iterative development and refinement of SPIN into a digital working prototype of SPIN, ready for implementation and trialling. There will be a large amount of collaborative work between the participants, computer engineer, web-developer, stakeholder advisory group and the researcher during this phase. 


\section{Participants}

Up to 10 participants who originally participated in the planning phase will be purposefully sampled to take part in the focus groups. Up to five of those participants will then be further purposefully sampled to be involved in individual think-aloud sessions. ${ }^{38}$ Sampling will aim for a range of abilities and levels of comfort when interacting with the prototype. Sample size is based on previous usability work by Nielsen ${ }^{39}$ and Virzi. ${ }^{40}$

\section{Data collection}

Focus groups will use wireframes as a prompt for discussion. The facilitator will lead exploration of the wireframes and will use exercises to elicit feedback on the intervention features, layout, order and content. ${ }^{33}$ For example, initial discussion will be generated around broad topics including initial impressions, including positive and negative feedback. More specific exercises will ask participants to sort prototype features in order of preference and importance. Sessions will be audio and video recorded with notes taken during the process.

Focus group findings will lead to development of a working digital prototype. Following that, individual think-aloud sessions will be used to gain a more in-depth, real-time understanding of how easy the working prototype is to use and follow and how participants interact with the intervention and progress through the stages. In these sessions, we will invite participants to work through a 'live' component of the prototype (eg, one exercise). They will be encouraged to speak their thoughts out loud while performing the representative task, commenting on what they are looking at, thinking, doing and feeling at each moment, in as close to their natural environment as possible. ${ }^{41}$ These sessions will be audio and video recorded. Open-ended interviews and postexperience questionnaires may also be used. ${ }^{41}$

\section{Data analysis}

Audio recordings from focus groups and think-aloud sessions will be transcribed verbatim. Data will be analysed using a directed content approach, ${ }^{34}$ drawing on usability frameworks. ${ }^{42}$ Transcripts will be read and key concepts relevant to preidentified usability elements will be highlighted. Consistent with directed content analysis, text that does not fit an existing code will be given a new code. ${ }^{34}$ Data from this phase will be used to refine the SPIN prototype in preparation for implementation and trialling (outside the scope of the current proposed research).

\section{Future work}

Should findings from the intervention's design and development phases indicate the website is acceptable and usable, we will progress to a mixed methods pilot trial of a 12-week SPIN intervention. The aim of this pilot trial will be to explore the feasibility, acceptability, safety and engagement of the intervention. The full protocol will be informed by the framework proposed by Proudfoot et at ${ }^{43}$ outlining facets, elements and guidelines of best practice in evaluating and reporting internet interventions. ${ }^{44}$ The full scope of this pilot trial will depend on findings from earlier phases. It is anticipated that up to 10 people with SCI who have not been involved with any of the intervention development phases will be invited to take part. Data such as pain and adverse events will be collected concurrently. Data such as shoulder pain and function will be collected pre and post intervention. Post-trial interviews will explore user experiences on the acceptability and the perceived benefits of the SPIN intervention.

\section{CONCLUSION}

This paper has described how we plan to develop a selfguided, web-based, exercise intervention (SPIN) to treat shoulder pain in people living with SCI. Using the PBA involves people living with SCI at each phase, increasing the likely engagement and effectiveness of the planned intervention.

\section{DISSEMINATION}

The results of this study will be published in a peer-reviewed journal and presented at relevant national and international conferences. A summary of findings will be presented to key stakeholder groups. The findings will also underpin the planned implementation and trialling phase, which will be the subject of future related research.

Acknowledgements The study's concept and aspects of it have previously been presented in poster format at the American College of Rehabilitation Medicine conference in 2017 and are published as a conference abstract (Stavric et al, Arch Phys Med Rehab 2017;98:e170-e170).

Contributors Concept of the project was devised by all authors. VS wrote the first and subsequent drafts of this manuscript. NS and NMK contributed and commented on it. NS and NMK advised on the study design and data analysis. This manuscript has been read and approved by all authors. All persons listed as authors have contributed to preparing the manuscript and the ICMJE criteria for authorship have been met. No other person other than the authors listed have contributed significantly to its preparation. The content of this manuscript is our original work and has not been published in whole either prior to or simultaneously with our submission to BMJ Open.

Funding This work was published with the support of the Centre for PersonCentred Research, Auckland University of Technology, New Zealand. The research described in this protocol is being supported by funding from AUT University, Physiotherapy New Zealand and the Neurology Special Interest Group of Physiotherapy New Zealand.

Competing interests None declared.

Patient consent for publication Not required.

Ethics approval Ethical approval has been granted by the Auckland University of Technology Ethics Committee (AUTEC).

Provenance and peer review Not commissioned; externally peer reviewed.

Open access This is an open access article distributed in accordance with the Creative Commons Attribution Non Commercial (CC BY-NC 4.0) license, which permits others to distribute, remix, adapt, build upon this work non-commercially, and license their derivative works on different terms, provided the original work is properly cited, appropriate credit is given, any changes made indicated, and the use is non-commercial. See: http://creativecommons.org/licenses/by-nc/4.0/. 


\section{REFERENCES}

1. Dalyan M, Cardenas DD, Gerard B. Upper extremity pain after spinal cord injury. Spinal Cord 1999;37:191-5.

2. Gutierrez DD, Thompson L, Kemp B, et al. The relationship of shoulder pain intensity to quality of life, physical activity, and community participation in persons with paraplegia. J Spinal Cord Med 2007;30:251-5

3. McCasland LD, Budiman-Mak E, Weaver FM, et al. Shoulder pain in the traumatically injured spinal cord patient: evaluation of risk factors and function. J Clin Rheumatol 2006;12:179-86.

4. Pentland WE, Twomey LT. Upper limb function in persons with long term paraplegia and implications for independence: Part I. Spinal Cord 1994;32:211-8

5. Sie IH, Waters RL, Adkins RH, et al. Upper extremity pain in the postrehabilitation spinal cord injured patient. Arch Phys Med Rehabil 1992;73:44-8.

6. Curtis KA, Drysdale GA, Lanza RD, et al. Shoulder pain in wheelchair users with tetraplegia and paraplegia. Arch Phys Med Rehabil 1999;80:453-7.

7. Curtis KA, Tyner TM, Zachary L, et al. Effect of a standard exercise protocol on shoulder pain in long-term wheelchair users. Spinal Cord 1999;37:421-9.

8. Nash MS, van de Ven I, van Elk N, et al. Effects of circuit resistance training on fitness attributes and upper-extremity pain in middle-aged men with paraplegia. Arch Phys Med Rehabil 2007;88:70-5

9. Nawoczenski DA, Ritter-Soronen JM, Wilson CM, et al. Clinical trial of exercise for shoulder pain in chronic spinal injury. Phys Ther 2006;86:1604-18.

10. Van Straaten MG, Cloud BA, Morrow MM, et al. Effectiveness of home exercise on pain, function, and strength of manual wheelchair users with spinal cord injury: a high-dose shoulder program with telerehabilitation. Arch Phys Med Rehabil 2014;95:1810-7.

11. Mulroy SJ, Thompson L, Kemp B, et al. Strengthening and optimal movements for painful shoulders (STOMPS) in chronic spinal cord injury: a randomized controlled trial. Phys Ther 2011;91:305-24.

12. Cratsenberg KA, Deitrick CE, Harrington TK, et al. Effectiveness of exercise programs for management of shoulder pain in manual wheelchair users with spinal cord injury. J Neurol Phys Ther 2015;39:197-203.

13. Ditor DS, Latimer AE, Ginis KAM, et al. Maintenance of exercise participation in individuals with spinal cord injury: effects on quality of life, stress and pain. Spinal Cord 2003;41:446-50.

14. Burkell JA, Wolfe DL, Potter PJ, et al. Information needs and information sources of individuals living with spinal cord injury. Health Info Libr J 2006;23:257-65.

15. Cowan RE, Nash MS, Anderson KD. Exercise participation barrier prevalence and association with exercise participation status in individuals with spinal cord injury. Spinal Cord 2013;51:27-32.

16. Griffiths F, Lindenmeyer A, Powell J, et al. Why are health care interventions delivered over the Internet? A systematic review of the published literature. J Med Internet Res 2006;8:e10.

17. Coulter EH, McLean AN, Hasler JP, et al. The effectiveness and satisfaction of web-based physiotherapy in people with spinal cord injury: a pilot randomised controlled trial. Spinal Cord 2017;55:383-9

18. Shepherd Centre. SCI-Ex exercise APP 2017.

19. Michie S, Yardley L, West R, et al. Developing and evaluating digital interventions to promote behavior change in health and health care: recommendations resulting from an international workshop. J Med Internet Res 2017;19:e232.

20. Yardley L, Morrison L, Bradbury K, et al. The person-based approach to intervention development: application to digital health-related behavior change interventions. J Med Internet Res 2015;17:e30.

21. Michie S, van Stralen MM, West R. The behaviour change wheel: a new method for characterising and designing behaviour change interventions. Implementation Sci 2011;6.
22. Band R, Bradbury K, Morton K, et al. Intervention planning for a digital intervention for self-management of hypertension: a theory-, evidence- and person-based approach. Implementation Sci 2017;12.

23. Webb TL, Joseph J, Yardley L, et al. Using the Internet to promote health behavior change: a systematic review and meta-analysis of the impact of theoretical basis, use of behavior change techniques, and mode of delivery on efficacy. J Med Internet Res 2010;12:18.

24. Morrison LG, Yardley L, Powell J, et al. What design features are used in effective e-health interventions? A review using techniques from critical interpretive synthesis. Telemedicine and e-Health 2012;18:137-44.

25. Burwood Academy of Independent Living. Our consultation services no date. Available: https://www.burwood.org.nz/research/ourconsultation-services/ [Accessed 12 Apr 2019].

26. Moher D, Liberati A, Tetzlaff J, et al. Preferred reporting items for systematic reviews and meta-analyses: the PRISMA statement. PLoS Med 2009;6:e1000097.

27. Higgins JPT, Altman DG, Gøtzsche PC, et al. The Cochrane collaboration's tool for assessing risk of bias in randomised trials. BMJ 2011;343:d5928.

28. Critical Appraisal Skills Programme. CASP randomised controlled trial checklist, 2019. Available: https://casp-uk.net/casp-toolschecklists/

29. Critical Appraisal Skills Programme. CASP qualitative checklist 2019. Available: https://casp-uk.net/casp-tools-checklists/

30. Thorne S, Kirkham SR, MacDonald-Emes J. Interpretive description: a noncategorical qualitative alternative for developing nursing knowledge. Res Nurs Health 1997;20:169-77.

31. Malterud K, Siersma VD, Guassora AD. Sample size in qualitative interview studies: guided by information power. Qual Health Res 2016;26:1753-60.

32. Turner D. Qualitative interview design: a practical guide for novice Investigators. Qual Rep 2010;15:754-60.

33. Caplan S. Using focus group methodology for ergonomic design. Ergonomics 1990;33:527-33.

34. Hsieh H-F, Shannon SE. Three approaches to qualitative content analysis. Qual Health Res 2005;15:1277-88.

35. QSR international Pty Ltd. NVivo qualitative data analysis software version 12. Doncaster; 2018.

36. Thorne S. The art (and science) of critiquing qualitative research. In: Morse JM, ed. Completing a qualitative project: details and dialogue. California, USA: Sage Publications, Inc, 1997.

37. Baier RR, Cooper E, Wysocki A, et al. Using qualitative methods to create a home health web application user interface for patients with low computer proficiency. EGEMS 2015;3.

38. Jaspers MWM, Steen T, van den Bos C, et al. The think aloud method: a guide to user interface design. Int $J$ Med Inform 2004;73:781-95.

39. Nielsen J. Why you only need to test with 5 users, 2000. Available: https://www.nngroup.com/articles/why-you-only-need-to-test-with5-users/

40. Virzi RA. Refining the test phase of usability evaluation: how many subjects is enough? Hum Factors 1992;34:457-68.

41. Georgsson M, Staggers N. An evaluation of patients' experienced usability of a diabetes mHealth system using a multi-method approach. J Biomed Inform 2016;59:115-29.

42. Nielsen J. 10 usability heuristics for user interface design: Nielsen Norman group, 1994. Available: https://www.nngroup.com/articles/ ten-usability-heuristics/ [Accessed 28 Mar 2018]

43. Proudfoot J, Klein B, Barak A, et al. Establishing guidelines for executing and reporting Internet intervention research. Cogn Behav Ther 2011;40:82-97.

44. Eysenbach G, CONSORT-EHEALTH Group. CONSORT-EHEALTH: improving and standardizing evaluation reports of web-based and mobile health interventions. J Med Internet Res 2011;13:e126-34. 